\title{
Produção científica sobre nutrição no âmbito da Atenção Primária à Saúde no Brasil: uma revisão de literatura
}

\author{
Scientific output on nutrition in the scope of Primary Health \\ Care in Brazil: a review of the literature
}

Daniela Silva Canella ${ }^{1}$

Ana Carolina Feldenheimer da Silva ${ }^{1}$

Patrícia Constante Jaime ${ }^{1}$

${ }^{1}$ Departamento de N utrição, FaculdadedeSaúdePública, Universidade de São Paulo. Avenida Dr Arnaldo 715/2응 Cerqueira Cesar. 01246-904 São Paulo SP. dcanella@usp.br
Abstract N utrition campaignsin Primary H ealth Care (PHC) play an important role in health promotion and the prevention and treatment of injuries. The scope of this paper is to chart and evaluate the scientific output of nutrition in Brazilian PHC. A search and review of the literature and papers was conducted on the PubM ed and Lilacs databases, using key words related to PH C and nutrition. Thestudies were restricted to Brazil with the professionals or population assisted by PHC in the Brazilian U nified Health System and published prior to $M$ arch 2011. The references in the selected articles were also consulted in order to identify additional studies. From the total of papers located, 68 were eligibleand a further 49 were identified in the references lists, such that a total of 117 paperswereanalyzed. Thestudies reviewed were mostly original articles, using quantitative methodology, carried out by São Paulo U niversity in that stateand published from 2002 to 2011. Themain issues were diagnosis seeking the evaluation of nutritional status involving children. The output in this field is growing, although there is a need to redirect the scope of future studies to a focus on intervention models and program evaluation.

Key words Primary health care, Family health, Primary health units, Nutrition in public health, N utritional assessment, Literature review as a topic
Resumo Introdução: A ções denutrição na Atenção Primária à Saúde (APS) têm importante papel na promoção da saúdee na prevenção e tratamento de agravos. Objetivo: M apear, si stematizar eavaliar a produção científica da nutrição no âmbito da APS no Brasil. Métodos: Trata-se de uma revisão de literatura. A busca de artigos foi feita no PubM ed e Lilacs, utilizando descritores referentes à APS e à nutrição. Foram levantados estudos conduzidos no Brasil, com profissionais ou população atendida pelo SU S na APS e publicados até março de 2011. Ainda, foram consultadas as referências dos artigos selecionados, a fim de identificar outros estudos. Resultados: $\mathrm{D}$ o total de artigos localizados, 68 foram elegíveis e 49 foram identificados nas listas de referências, totalizando 117 artigos analisados. Estes foram, em sua maioria, artigos originais, quantitativos, conduzidosno estado deSP, pela USP epublicados de 2002 a 2011. Abordavam o diagnóstico, tendo como objeto a avaliação do estado nutricional e envolviam crianças. Conclusão: A produção na área é crescente, porém há necessidade de redirecionar a abordagem e o objeto de futuros estudos, focando em modelos de intervenção e em avaliação de programas.

Palavras-chave A tenção Primária à Saúde, Saúde da família, U nidades Básicas de Saúde, N utrição em saúde pública, Avaliação nutricional, Literatura de revisão como Assunto 
I ntrodução

O conceito de Atenção Primária à Saúde (APS) decorre de 1978, da Declaração de Alma Ata, e compreende-se que esta écomposta de cuidados essenciais de saúde baseados em métodos, tecnologias práticas, evidências científicas os quais devem ser socialmente aceitáveis, e estar ao alcance universal de indivíduos e famílias da comunidade, mediante sua plena partici pação ${ }^{1}$. No Brasil, a APS écaracterizada por um conjunto de ações, de âmbito individual e coletivo, situadas no primeiro nível deatenção nos sistemas desaúde, que abrange promoção e proteção da saúde, prevenção de agravos, diagnóstico, tratamento, reabilitação e manutenção da saúde ${ }^{2}$. Desde sua origem, em 1994, o Programa de Saúde da Família (PSF), posteriormente denominado Estraté gia Saúde da Família (ESF), foi concebido como uma estratégia para a reorganização e fortalecimento da atenção básica como o primeiro nível de atenção à saúde no SUS, por meio da ampliação do acesso, da qualificação e da reorientação das práticas de saúde ${ }^{1}$. Desta forma, a APS se fortaleceu de forma gradual, sendo, atualmente, a porta de entrada preferencial para o Sistema Ú nico de Saúde (SUS)².

No âmbito da APS no país, as ações de alimentação enutrição e do cuidado nutricional estão inseridas na aten ção à saúde e compreendem atividades como a vigilância al imentar enutricional, promoção da alimentação saudável e programas de prevenção e controle de distúrbios nutricionais, tais como anemia ferropriva ehipovitaminoseA, além do acompanhamento dascondicionalidades de programas governamentais, como o Programa Bolsa Família. Atualmente, tais ações são sistematizadas e organizadas na $M$ atriz de Ações de Alimentação e Nutrição na Atenção Básica de Saúde, representando um esforço convergentee complementar às ações quejá vêm sendo implementadas por diversos programas públicos de saúde, como a Saúde da Família?.

Em relação à nutrição na APS, estudo conduzido no Canadá aponta que um modelo deserviço de nutriçãa interdisciplinar, com diferentes e complementares serviços de apoio, é mais prático e acessível à população, com melhor custoefetividadeesustentabilidade do que um modelo de serviço de nutrição não interdisciplinar ${ }^{4}$.

Assim, entendendo queas ações de al imentação enutrição vão além das atividades desempe nhadas por nutricionistas e tem importante papel na promoção da saúde da população e na prevenção e tratamento de agravos, e tendo em vista a ausência de revisões envolvendo esta temática, a presente teve como objetivo mapear, sistematizar e avaliar a produção científica da nutrição na Atenção Primária à Saúde no Brasil, a fim de contribuir para a reflexão de um possível redirecionamento de futuros estudos na área, no que diz respeito a sua abordagem.

\section{Métodos}

Realizou-se uma revisão de literatura, tendo como pergunta: Como se caracteriza a produção científica da nutrição na Atenção Primária à Saúde no Brasil?

Critérios de inclusão e exclusão de estudos

Foram levantados estudos real izados no Brasil, tendo como sujeitos a população atendida pelo SU S no nível de atenção primária, independente do ciclo da vida a que pertencem eda condição de saúde (presença de patologia ou fator de risco), e, ainda, os profissionais de saúde que atuam no SUS e, publicados até março de 2011, nos idiomas português, inglês ou espanhol.

Os estudos que não envolviam o SUS, que tinham como foco outros níveis de atenção que não a primária, não envolviam a alimentação e nutrição epublicações deoutra natureza, quenão eram artigos, foram excluídos.

\section{Estratégia de busca e sel eção de estudos}

A estratégia de busca dos estudos foi dese nhada para ser realizada na base de dados PubM ed e adaptada para a busca no Lilacs.

Considerou-se os seguintes limites de busca: estudos em humanos, publicados em português, inglês ou espanhol e com data limite de publicação em março de 2011. Dois conjuntos de intersecção de termos de busca bibliográfica foram combinados: atenção primária à saúde [atenção primária à saúde / cuidados primários de saúde / atenção básica à saúde ( primary health care), saúde da família (family health), centros de saúde/unidades básicas de saúde (community health centers), serviços de saúde (health services) ] e nutrição [dieta (diet), estado nutricional (nutritional status), consumo alimentar (food consumption), hábito alimentar (food habits), antropometria (anthropometry, anthropometry measurements), avaliação nutricional (nutritional assessment)]. Cada termo foi cruzado individualmente com outro, de forma que garantisse a inclusão de to- 
dos os artigos relacionados ao tema. No caso do PubM ed, foi incluído na busca o termo Brasil (Brazil), o mesmo não sendo feito para o Lilacs, já que este conta com grande volume de produção local e a inclusão do termo poderia limitar excessivamente a busca. Foram pesquisadas também as listas de referência dos artigos, a fim de identificar estudos relevantes não identificados na busca.

Como primeira avaliação, foram observados o título e o resumo dos artigos, sendo rejeitados aqueles que não atendiam aos critérios de inclusão ou apresentavam algum critério de exclusão. N os casos de incerteza, o texto completo foi consultado para confirmar a elegibilidade do estudo.

\section{Síntese e comparação de estudos}

Os artigos foram sistematicamente revisados em relação à sua adequação ao tema e, posteriormente os estudos foram apresentados de forma descritiva, com base em classificação, segundo categorias propostas, por meio de frequências absolutas e relativas.

As categorias analíticas foram estipuladas no planejamento da revisão e organizadas para compilação dos dados. 0 resultado dessa sistematização gerou oito categorias: Tipo de estudo (original, revisão, opinião); M étodo (quantitativo, qualitativo, misto ou não se aplica); Local (onde o estudo foi desenvolvido); Vínculo institucional (instituição responsável por sua condução); Ano de publicação; N atureza da abordagem (diagnóstico, determinantes, terapêutica / protocolo de atendimento / modelo de intervenção, relato de experiência, avaliação de programas e serviços, vigilância / monitoramento, histórico); O bjeto (carências nutricionais, estado nutricional, doenças crônicas não transmissíveis, segurança alimentar enutricional, promoção da saúde/ alimentação saudável/ educação em saúde, gestão e financiamento, consumo alimentar, al eitamento materno, serviços de saúde, Programa Bolsa Família ou Bolsa Alimentação); Sujeitos (referente ao ciclo de vida estudado, a indivíduos com determinada patologia ou fator de risco, ou, ainda, a profissionais de saúde trabalhadores do SUS).

\section{Resultados}

$\mathrm{Na}$ estratégia de busca foram identificados 302 artigos, não duplicados, dos quais 233 não atendiam aos critérios de elegibilidade, resultando em 68 artigos para análise. Posteriormente, outros
49 estudos foram localizados a partir das listas de referências dos artigos sel ecionados, totalizando 117 artigos a serem analisados (Figura 1).

Nabusca inicial em basededados, o principal motivo para a exclusão de documentos foi a não adequação ao tema da investigação, sendo recorrente encontrar trabalhos que envolviam outros níveis de assistência à saúde que não a atenção primária ( $n=78$ ), estudos que não tratavam de atenção à saúde e que não foram desenvolvidos no âmbito do SUS $(n=52)$, além de 37 documentos localizados que não eram artigos científicos e sim teses, livros, anais de congressos, documentos técnicos e manuais (Figura 1).

O processo completo de seleção dos artigos pode ser visto na Figura 1.

Os documentos identificados foram, prioritariamente, artigos originais ( $n=113$ ), envolvendo o método deanálise quantitativo $(n=104)$ e publicados na última década ( $n=75$ ). Estes foram real izados em 13 estados, local izados, principalmente, nas regiões Sudestee Sul do país, sendo os estados alvo da maioria dos estudos São Paulo ( $n=38)$, Rio de Janeiro $(n=16)$ e Rio Grande do Sul ( $n=15$ ). Os estudos localizados foram conduzidos por 39 instituições, sendo a

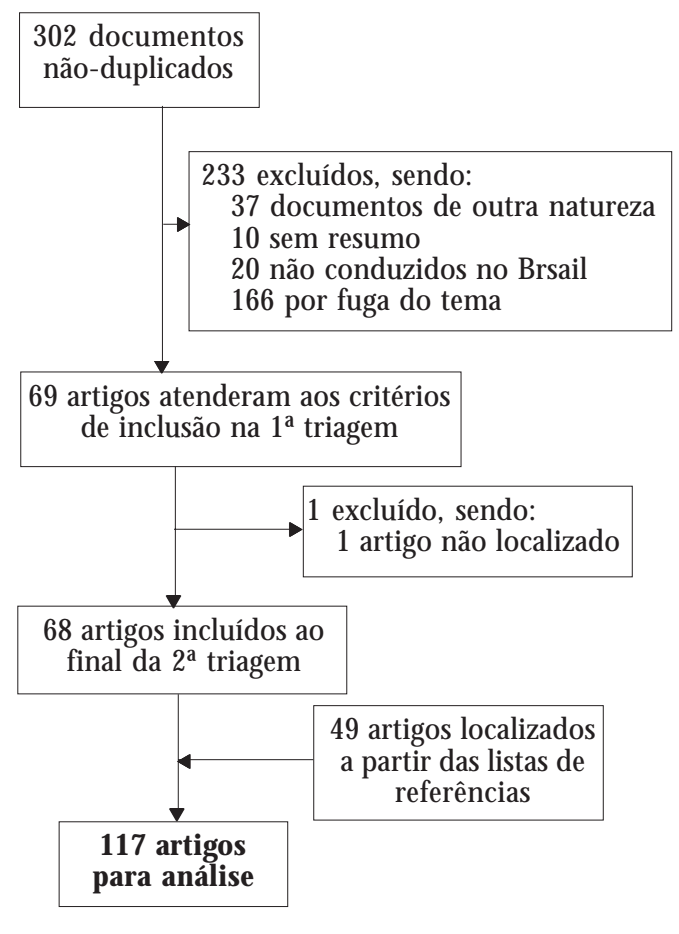

Figura 1. Descrição da seleção dos estudos. 
maior parte sob a responsabilidade da Universidade de São Paulo ( $n=20)$, da Universidade Federal do Rio Grande do Sul ( $n=11$ ) e da UniversidadeFederal de São Paulo $(n=8)$ (Tabela 1$)$.

Estudos de revisão da literatura sobre a temática não foram recuperados nas buscas em bases de dados e em listas de referências (Tabela 1).

$\mathrm{N}$ o que se refere à abordagem e ao objeto de estudo, os artigos estão voltados, ainda prioritariamente, à realização de diagnóstico $(n=53)$, com foco na avaliação do estado nutricional ( $n$ =43). No entanto, nota-se um aumento na produção referente à terapêutica, protocolos de atendimento e modelos de intervenção $(n=25)$, bem como estudos focados em doenças crônicas não transmissíveis (DCNT) ( $n=24)$. As crianças ainda são os sujeitos mais estudados ( $n=$ $45)$, porém adultos $(n=32)$ e gestantes $(n=25)$, tem recebido alguma atenção. D estaca-se que no grupo dos adultos, sete estudos se referiam exclusivamente a mulheres, sendo estas no pósparto, lactantes ou mães (Tabela 2).

Estudos que tivessem como objeto a segurança alimentar e nutricional (SAN) e programas como Bolsa Família ou Bolsa Alimentação não foram localizados nas buscas.

\section{Discussão}

Com base nos achados desta revisão de literatura, pode-se afirmar que as pesquisas e, conse quentemente, o volume de publicações referente à nutrição na APS vem crescendo, porém o foco destes estudos merece ser discutido.

Localizou-se um elevado número de artigos sobre a temática, no entanto, do total de trabaIhos analisados, mais de $95 \%$ se referem a artigos originais, sendo que nenhuma revisão de literatura foi encontrada. Tal fato pode estar relacionado à grande diversidade de objetivos, desenhos deestudos edesfechos estudados no nível da atenção primária, ou a uma possível baixa qualidade destes estudos, o que dificulta a condução de re visão sistemática ou metanálise que possa gerar evidências clínica e em saúde pública ${ }^{5,6}$. Os artigos de opinião, apesar de quatro terem sido localizados, de fato, são escassos na literatura em nutrição ${ }^{7}$.

Em relação ao método utilizado, verificou-se a predominância pelo quantitativo (cerca de $90 \%$ dos estudos), refletindo o que ocorre, de forma geral, nas pesquisas na área da saúde, tendo em vista a necessidade de quantificar eventos, causas e efeitos ${ }^{8}$, por vezes ignorando a complexidade dosfenômenos relacionados à saúde pública, que deveriam considerar a complementaridade dos métodos quantitativos e qual itativos, e não a dicotomia entre estes ${ }^{9}$. No entanto, vale ressaltar que dos oito estudos encontrados que empregavam o método qualitativo, $75 \%$ foram publicados a partir do ano de2002, apontando para um crescimento nestetipo de estudo.

Historicamente, o M inistério da Saúde (M S) desempenhou um papel secundário no fomento à pesquisa em saúde, apesar da criação do SU S já em 1988. Tal negligência resultou em um distanciamento entre a produção científica e as reais necessidades de saúde da população. A partir do ano 2000 um novo cenário começou a ser dese nhado, com a criação do Departamento de Ciência e Tecnologia (DECIT) ${ }^{10}$. Segundo dados do portal Pesquisa Saúde, do M inistério da Saúde ${ }^{11}$, no período de 2002 a 2010, cerca de 13 milhões de reais foram concedidos em financiamento a 247 projetos de pesquisa do campo da Alimentação e Nutrição. Isto pode justificar grande parte da produção científica no período de 2002 a 2011, que corresponde a quase $65 \%$ dos artigos localizados.

No que se refere ao local de realização dos estudos e ao vínculo institucional das pesquisas conduzidas, observa-se que o estado de São Paulo e a Universidade de São Paulo, respectivamente, se destacam no país. Na última década, tal achado tem forte relação com o número de financiamentos concedidos pelo M S a este estado, bem como a esta Universidade, em específico ${ }^{11}$. Ainda, o estado de São Paulo conta, desde a dé cada de 1960, com uma forte agência de fomento, a Fundação de Amparo à Pesquisa do Estado de São Paulo (FAPESP), o que também tem influência sobre sua produção científica ${ }^{12}$.

Outros estados e Universidades com grande número de projetos financiados são Rio de Janeiro, M inas Gerais e Rio Grande do Sul, e Universidade Federal da Bahia (UFBA), UniversidadeFederal do Rio de Janeiro (UFRJ), Universidade de M inas Gerais (UFM G) e Universidade Federal do Rio Grande do Sul (UFRGS), respectivamente ${ }^{11}$. Exceto por M inas Gerais, pelaUFBA eUFM G, os demais estados eUniversidades apre sentam um volume de publicação coerente com o recebimento derecursos. Destaforma, o quese observa é uma forte desigualdade regional nos investimentos em pesquisa, com os recursos concentrados nas regiões Sudeste e Sul. No entanto, a diferença nos investimentos em pesquisa entre as regiões do país não é vista de forma isolada. Em relação ao financiamento federal do SUS, por 
anos, o que se observou foi uma alocação desigual de recursos, sendo a maior parte destinada às regiões mais desenvolvidas do país, coincidentemente Sudestee Sul ${ }^{13}$.

Porém, merece menção que, a partir de 2004, com a Política N acional de Ciência, Tecnologia e Inovação em Saúde, tem-se como estratégia a orientação do fomento segundo prioridades, com criação de mecanismos para a superação das desigual dades regionais se destaque ao financiamento de projetos e a coordenação e realização de pesquisas em regiões menos desenvolvidas do País, às quais devem ser destinados $30 \%$ do financiamento total de cada editall ${ }^{14}$. Tal fato, pode resultar em melhor distribuição da produção em alimentação e nutrição no futuro.

A pesar deste não ser o foco das análises, vale mencionar que o grande número de artigos não localizados nas buscas em bases de dados, mas incluídos na revisão, a partir da consulta às listas de referências, deve-se, em partes, à escolha inadequada de palavras-chave por parte dos autores dos artigos, uma vez que muitas destas não correspondem aos Descriptores de Ciencias de la Salud (DeCS) ou M edical Subject H eadings (M eSH).

Posterior à criação do SUS e integrando a Política Nacional deSaúde, foi elaborada a Política N acional deAlimentação e N utrição (PNAN), de 1999. Entresuas sete diretrizes programáticas, destaca-se uma que trata da promoção do desenvolvimento de linhas de investigação, na qual se sinaliza a importância eo apoio a pesquisas relacionadas à desnutrição energético-proteica; deficiência de micronutrientes; relação entre DCNT e dieta; consumo e padrão alimentar; composição e valor nutritivo de al imentos e preparações culinárias; composição alimentar e nutricional das refeições oferecidas em grandes quantidades, por exemplo, no Programa de Alimentação do Trabalhador; aleitamento materno e transição para a alimentação complementar; pesquisas de natureza antropológica e etnográfica sobre hábitos e práticas alimentares; além da proposição, avaliação e validação de modelos de intervenção, considerando eficácia, efetividade e custo-benefício ${ }^{15}$.

Com base nas linhas de pesquisa prioritárias expostas pela primeira edição da PN AN é possível perceber o cenário epidemiológico de transição nutricional no qual esta foi construída ${ }^{15}$. Percebe-se, ainda, como este cenário se reflete de maneira significante na condução dos estudos localizados, os quais apresentam forte interesse em realizar o diagnóstico e o monitoramento do estado nutricional da população, prioritariamen- te de crianças, que são um clássico grupo de risco. No entanto, em virtude da globalização, da comensalidade contemporânea ${ }^{16,17}$, do crescente aumento na prevalência de excesso depeso eobesidade $^{18}$, e, concomitantemente, das DCNT associadas a esta condição, o volume de publicações referentes a DCNT econsumo alimentar vem aumentando.

Da implantação da PNAN aos dias de hoje podem ser destacados como alguns de seus mé ritoso desenvolvimento da vigilância alimentar e nutricional, a produção regular de informações sobre estado nutricional da população e a construção da agenda de promoção da alimentação saudável ${ }^{19}$, o que é coerente com a direção e 0 foco que as pesquisas sobre a nutrição no âmbito da APS têm tido. No entanto, há a necessidade de maior incentivo ao desenvolvimento e à publicação, ainda que os resultados sejam negativos, deestudos queavaliem a terapêutica, os protocolos de atendimento e os modelos de intervenção desenvolvidos no nível primário de atenção à saúde, bem como a realização de avaliações de processo e de impacto de programas e serviços de saúde locais, regionais e nacionais, como o Bolsa Família. M encionando novamente a PNAN, ainda que estetipo deavaliação seja apontado como uma linha deinvestigação prioritária aparece de forma secundária na Política.

No tocanteaos sujeitos estudados, aindahoje as crianças constituem um grupo privilegiado, no entanto, gestantes, adultos, idosos e indivíduos com determinada patologia também têm sido alvo de investigações. É necessário que as ações em saúde e, consequentemente, mais estudos priorizem os adolescentes, principalmente com o olhar para a atenção integral à sua saú$\mathrm{de}^{20}$. Ainda, a condução de estudos que avaliem a prática profissional, as percepções e a educação permanente de profissionais de saú de da APS são de grande relevância.

Conclui-se que produção na área écrescente, porém a maioria dos estudos, ainda realizados nas regiões Sudeste e Sul do país, limitou-se ao diagnóstico de populações específicas, principalmente a infantil, utilizando a avaliação do estado nutricional. Os estudos relacionados à avaliação de programas, no geral, não tratam do impacto, mas apenas de sua implementação. Assim, esta revisão da literatura aponta para a necessidade de redirecionamento das pesquisas relacionadas à nutrição no âmbito da APS no Brasil. No que tange aos financiamentos públicos para pesquisa, os órgãos de fomento devem atentar para uma distribuição igualitária ou equitativa dos 
Tabela 1. Classificação dos artigos incluídos na revisão, segundo características dos documentos levantados.

\begin{tabular}{|c|c|c|c|c|}
\hline Categorias analíticas & $\begin{array}{l}\text { Base de } \\
\text { dados } \\
\text { N }\end{array}$ & $\begin{array}{l}\text { Lista de } \\
\text { referências } \\
\mathrm{N}\end{array}$ & $\begin{array}{c}\text { Total } \\
\mathrm{N}(\%)\end{array}$ & Referências \\
\hline \multicolumn{5}{|l|}{ Tipo de estudo } \\
\hline Original & 67 & 46 & $113(96,6)$ & $21-133$ \\
\hline Opinião & 1 & 3 & $4(3,4)$ & 134- 137 \\
\hline \multicolumn{5}{|l|}{ M étodo } \\
\hline Quantitativo & 61 & 43 & $104(88,9)$ & $\begin{array}{l}21-41,43-47,50-63,65-68,70-82,84-93 \\
95-103,105-127,129-133\end{array}$ \\
\hline Qualitativo & 5 & 3 & $8(6,8)$ & $48,49,64,69,83,94,104,128$ \\
\hline Misto & 1 & 0 & $1(0,8)$ & 42 \\
\hline Não se aplica & 1 & 3 & $4(3,4)$ & $134-137$ \\
\hline \multicolumn{5}{|l|}{ Ano de publicação } \\
\hline 1977 a 1991 & 3 & 3 & $6(5,1)$ & $21-26$ \\
\hline 1992 a 2001 & 16 & 20 & $36(30,8)$ & $27-61,134,135$ \\
\hline 2002 a 2011 & 49 & 26 & $75(64,1)$ & 62-133, 136, 137 \\
\hline \multicolumn{5}{|l|}{ Local de realização (UF) } \\
\hline São Paulo & 19 & 19 & $38(32,5)$ & $\begin{array}{l}21-24,29,33-36,40,41,44,45,48-50,52 \\
56,59,64,65,67,70,73,74,77,79,88 \\
91,100,101,112,118,122,124,131-133\end{array}$ \\
\hline Rio de Janeiro & 8 & 8 & $16(13,7)$ & $\begin{array}{l}32,39,46,53,68,72,78,81-83,90,94 \\
98,106,117,120\end{array}$ \\
\hline Rio Grande do Sul & 11 & 4 & $15(12,8)$ & $\begin{array}{l}25,27,30,47,54,60,63,86,89,105 \\
115,125,128-130\end{array}$ \\
\hline Pernambuco & 5 & 3 & $8(6,8)$ & $55,84,92,96,102,109,113,114$ \\
\hline Bahia & 4 & 3 & $7(6,0)$ & $37,76,85,95,103,104,116$ \\
\hline Multicêntrico (6 capitais) & 5 & 1 & $6(5,1)$ & $43,51,57,58,61,126$ \\
\hline Brasil & 2 & 4 & $6(5,1)$ & $107,121,134-137$ \\
\hline Demais & 14 & 7 & $21(17,9)$ & $\begin{array}{l}26,28,31,38,42,64,66,69,71,75,80,87, \\
93,97,99,108,110,111,119,123,127\end{array}$ \\
\hline \multicolumn{5}{|l|}{$\begin{array}{l}\text { Vínculo institucional } \\
\text { (U niversidade /Instituição) }\end{array}$} \\
\hline USP & 10 & 10 & $20(17,1)$ & $\begin{array}{l}21,22,24,33,36,40,41,48,50,56,59 \\
64,67,74,91,99,102,112,131,133\end{array}$ \\
\hline UFRGS & 9 & 2 & $11(9,4)$ & $25,27,43,51,57,58,61,90,125,126,129$ \\
\hline UNIFESP & 6 & 2 & $8(6,8)$ & $35,44,45,73,79,93,122,132$ \\
\hline UFPel & 4 & 3 & $7(6,0)$ & $30,47,54,60,63,86,130$ \\
\hline UFRJ & 3 & 5 & $8(6,8)$ & $39,46,72,81,82,98,106,120$ \\
\hline UFPE & 3 & 1 & $4(3,4)$ & $92,102,109,114$ \\
\hline$|\mathrm{M}| \mathrm{P}$ & 2 & 4 & $6(5,1)$ & $55,84,96,113,134,135$ \\
\hline ENSP & 2 & 3 & $5(4,3)$ & $26,32,68,78,117$ \\
\hline Outras & 29 & 19 & $48(41,0)$ & $\begin{array}{l}23,28,29,31,34,37,38,42,49,52,53 \\
62,65,66,69-71,75-77,80,83,85,87- \\
89,94,95,97,100,103-105,107,108 \\
110,111,115,116,118,119,121,123 \\
124,127,136,137\end{array}$ \\
\hline
\end{tabular}

recursos entre as regiões e os estados brasileiros. Em relação à produção científica, éurgente a ne cessidade de maiores esforços para o delineamento e a condução de estudos que foquem em modelos de intervenção e na avaliação de progra- mas, o que já foi sinalizado em oficina de elaboração da subagenda de alimentação e nutrição, da Agenda Nacional de Prioridades de Pesquisa em Saúde, em 2010 (documento ainda não publicado). 


\begin{tabular}{|c|c|c|c|c|}
\hline Categorias analíticas & $\begin{array}{l}\text { Base de } \\
\text { dados } \\
\quad \mathrm{N}\end{array}$ & $\begin{array}{l}\text { Lista de } \\
\text { referências } \\
\quad \mathrm{N}\end{array}$ & $\begin{array}{l}\text { Total } \\
\text { N }(\%)\end{array}$ & Referências \\
\hline \multicolumn{5}{|l|}{ Natureza da abordagem } \\
\hline Diagnóstico & 30 & 23 & $53(45,3)$ & $\begin{array}{l}21-23,25,26,29,31,33,38-40,45-47,50- \\
52,54,59,62,63,66,67,69-71,74-78,81, \\
83,85,86,92,93,96,98,99,102,104,106, \\
109,111,116,124,125,129-131,133\end{array}$ \\
\hline D eterminantes & 3 & 2 & $5(4,3)$ & $80,97,110,102,120$ \\
\hline Vigilância/ monitoramento & 7 & 8 & $15(12,8)$ & $\begin{array}{l}27,37,56-58,72,79,82,88,100,108 \\
114,118,121,127\end{array}$ \\
\hline $\begin{array}{l}\text { Terapêutica/protocolo de } \\
\text { atendimento/modelo de } \\
\text { intervenção }\end{array}$ & 15 & 10 & $25(21,4)$ & $\begin{array}{l}24,28,34,36,41,43,44,48,49,53,55 \\
60,61,65,84,87,105,113,115,119 \\
123,126,132,134,135\end{array}$ \\
\hline $\begin{array}{l}\text { Avaliação de programas e } \\
\text { serviços }\end{array}$ & 10 & 6 & $16(13,7)$ & $\begin{array}{l}30,32,35,42,68,73,89-91,94,95,103 \\
107,117,122,136\end{array}$ \\
\hline Relato de experiência & 2 & 0 & $2(1,7)$ & 64,128 \\
\hline Histórico & 1 & 0 & $1(0,8)$ & 137 \\
\hline \multicolumn{5}{|l|}{ Objeto* } \\
\hline Estado nutricional & 26 & 17 & $43(36,8)$ & $\begin{array}{l}21-27,32,33,35,40-42,44-46,55-58,60 \\
62,65,72,73,75,81,82,87,89,95,96 \\
99,108,112,113,116,118-121,126,130\end{array}$ \\
\hline $\begin{array}{l}\text { Doenças crônicas não } \\
\text { transmissíveis }\end{array}$ & 18 & 6 & $24(20,5)$ & $\begin{array}{l}28,31,36,37,43,47,51,53,59,61,74 \\
86,88,90,91,100,103,105,123,124 \\
127,131-133\end{array}$ \\
\hline Carências nutricionais & 7 & 10 & $17(14,5)$ & $\begin{array}{l}29,39,50,55,66,75,79,80,84,93,107 \\
109,110,117,122,134,135\end{array}$ \\
\hline Consumo alimentar & 7 & 8 & $15(12,8)$ & $\begin{array}{l}65,67,69,71,76-78,83,85,92,98,102 \\
106,125,129\end{array}$ \\
\hline $\begin{array}{l}\text { Promoção da saúde/ } \\
\text { alimentação saudável/ } \\
\text { educação em saúde }\end{array}$ & 5 & 2 & $7(6,0)$ & $48,49,64,102,104,115,128$ \\
\hline Aleitamento materno & 3 & 8 & $11(9,4)$ & $30,34,38,46,52,68,70,77,94,97,111$ \\
\hline Serviços de saúde & 1 & 3 & $4(3,4)$ & $54,63,114,136$ \\
\hline Gestão e financiamento & 1 & 0 & $1(0,8)$ & 137 \\
\hline \multicolumn{5}{|l|}{ Sujeitos ${ }^{*}$} \\
\hline Gestantes & 16 & 9 & $25(21,4)$ & $\begin{array}{l}21,22,24,43,50,51,53,56-58,61,67 \\
71,78,84,96,102,108,112,115,120 \\
125,126,129,130\end{array}$ \\
\hline Crianças & 26 & 19 & $45(38,5)$ & $\begin{array}{l}23,25-27,29,33,35,38-42,44-46,52 \\
55,60,62,65,66,68,73,75,76,79,80 \\
85,89,93,95,97,99,104,107,110,111 \\
113,114,117,121,122,124,128,131\end{array}$ \\
\hline Adolescentes & 7 & 2 & $9(7,7)$ & $22,31,50,71,78,90,127$ \\
\hline Adultos & 18 & 15 & $32(27,4)$ & $\begin{array}{l}28,30,31,34,36,37,47,54,55,59,63 \\
64,70,72,74,77,81-83,86-88,90,91 \\
98,100,103,106,116,118,123,127,132\end{array}$ \\
\hline Idosos & 13 & 8 & $21(17,9)$ & $\begin{array}{l}28,31,36,37,47,59,74,86-88,90-92 \\
102,103,109,116,118,119,127,133\end{array}$ \\
\hline Indivíduos com DCNT & 11 & 9 & $20(17,1)$ & $\begin{array}{l}28,36,37,47,54,59,63,74,86-88,91 \\
100,103,105,118,119,123,127\end{array}$ \\
\hline Profissionais de saúde & 2 & 3 & $5(4,3)$ & $32,48,49,69,136$ \\
\hline Não se aplica & 1 & 3 & $4(2,3)$ & $94,134,135,137$ \\
\hline
\end{tabular}

${ }^{*}$ Alguns estudos envolvem mais de uma categoria 


\section{Colaboradores}

DS Canella participou da concepção e delineamento do estudo, bem como da análise dos dados e redação do artigo, ACF Silva participou da análise dos dados e revisão crítica do artigo e PC Jaime participou da concepção e delineamento do estudo e revisão crítica do artigo.

\section{Referências}

1. Sousa M F, Hamann EM. Programa Saúde da Família no Brasil: uma agenda incompleta? Cien Saude Colet 2009; 14(Supl. 1):1325-1335.

2. Brasil. Ministério da Saúde (MS). Secretaria de Atenção à Saúde. Departamento de Atenção Básica. Política Nacional de Atenção Básica. 4a Edição. Brasília: MS; 2007. (Série E. Legislação de Saúde).

3. Brasil. M inistério da Saúde (MS). Secretaria de Atenção à Saúde. Departamento de Atenção Básica. Matriz de ações de alimentação e nutrição na atenção básica de saúde. Brasília: M S; 2009. (Série A. Normas e $M$ anuais Técnicos).

4. Brauer P, Dietrich L, Davidson B. Nutrition in primary health care: using a Delphi process to design new interdisciplinary services. Can J Diet Prac Res 2006; 67(Supl.):S14-S29.

5. Cook D, Mulrow CD, Haynes RB. Systematic Reviews: synthesis of best evidence for clinical decisions. Ann Intern M ed 1997; 126(5):376-380.

6. Margetts B, Warm D, Yngve A, Sjöström M. Developing an evidence-based approach to Public Health Nutrition: translating evidence into policy. Public Health N utr 2001; 4(6A):1393-1397.

7. Tomás-Castera V, Sanz-Valero J, Wanden-Berghe C. Estudio bibliométrico de la producción científica de la Revista de Nutrição através de la Red SciELO (2001 a 2007). Rev Nutr 2010; 23(5):791-799.

8. Santos SR. M étodos qualitativos e quantitativos na pesquisa biomédica. J Pediatr 1999; 75(6):401-406.

9. M inayo MCS, Sanches O. Quantitativo-Qualitativo: O posição ou Complementaridade? Cad Saude Publica 1993; 9(3):239-262.

10. Serruya $S$, Vasconcellos AB. A Secretaria de Ciência e Tecnologia e Insumos Estratégicos do Ministério da Saúde e a pesquisa em alimentação e nutrição. Cad Saude Publica 2008; 24(Supl. 1):S361-S364.

11. Brasil. Ministério da Saúde. Pesquisa Saúde. [acessado 2011 jun 13]. Disponível em: http://pesquisa saude.saude.gov.br/bdgdecit/index.php?lg=BR

12. Brasil. São Paulo (Estado). Lei № 5.918, de 18 de outubro de 1960. Autoriza o Poder Executivo a instituir a "Fundação de Amparo à Pesquisa do Estado de São Paulo", e dá outras providências. Diário Oficial do Estado de São Paulo 1960; 18 out.

13. Souza RR. Redução das desigualdades regionais na alocação dos recursos federais para a saúde. Cien Saude Colet 2003; 8(2):449-460.
14. Brasil. Departamento de Ciência e Tecnologia, Secretaria de Ciência, Tecnologia e Insumos Estratégicos (DECIT). Ministério da Saúde. Pesquisa em Saúde no Brasil. Rev Saude Publica 2008; 42(4):773-775.

15. Brasil. M inistério da Saúde (M S). Secretaria de Atenção à Saúde. Departamento de Atenção Básica. Política Nacional de Alimentação e Nutrição. 2a Edição. Revisão. Brasília: M S; 2003. 48 p. (Série B. Textos Básicos de Saúde).

16. Garcia RWD. Reflexos da globalização na cultura alimentar: considerações sobre as mudanças na alimentação urbana. Rev Nutr 2003; 16(4):483-492.

17. Instituto Brasileiro de Geografia e Estatística (IBGE). Pesquisa de Orçamentos Familiares 2008-2009: avaliação nutricional da disponibilidade domiciliar de alimentos no Brasil. Rio de Janeiro: IBGE; 2010.

18. Instituto Brasileiro de Geografia e Estatística (IBGE). Pesquisa de Orçamentos Familiares 2008-2009: antropometria e estado nutricional de crianças, adolescentes e adultos no Brasil. Rio de Janeiro: IBGE; 2010.

19. Recine $E$, Vasconcellos AB. Políticas nacionais e o campo da Alimentação e Nutrição em Saúde Coletiva: cenário atual. Cien Saude Colet 2011; 16(1):73-79.

20. Horta NC, Sena RR. Abordagem ao adolescente e ao jovem nas políticas públicas de saúde no Brasil: um estudo de revisão. Physis 2010; 20(2):475-495.

21. Siqueira AAF, Ciari Júnior $C, M$ attos ILB, Buralli KO, Batista Filho M, Schor N, Almeida PAM, d'Andretta Tanaka AC. Aplicação de uma curva de ganho de peso para gestantes. Rev Saude Publica 1977; 11(2):288-293.

22. Siqueira AA, Tanaka AC, Andrade J, Almeida PA, Santiago S, Zanatelli CC, Bier R, Queiros BM, Saran MR. Evolução da gravidez em adolescentes matriculadas no Serviço Prénatal do Centro de Saúde Geraldo de Paula Souza, São Paulo (Brasil). Rev Saude Publica 1981; 15(5):449-454.

23. Corrêa CEC, M ello M LOD, Anastácio M PD. Avaliação nutricional de 142 crianças de 0 a 14 anos, registradas no Centro de Saúde - Escola da Faculdade de Medicina de Catanduva: estudo transversal parcial. Klinikos 1985; 1(4):25-27.

24. M onteiro CA, Benicio MH, Lamonica IM, L'Abbate $\mathrm{S}$, Stefanini ML, O shiro JH, Bearzotti PH, Alves MCGP. Avaliação do impacto da suplementação alimentar a gestantes no controle do baixo peso ao nascer no município de São Paulo, SP (Brasil). Rev Saude Publica 1985; 19(5):458-474. 
25. Guigliani ERJ, Rotta AT, Ribeiro AM, M ello C, M oreira CM, Dias CCC, Prytaluk TM. Percepção materna sobre a adequação do peso e da altura de crianças menores de 5 anos em uma vila periférica de Porto Alegre. Rev HCPA 1990; 10:70-73.

26. Coimbra Júnior CEA, Santos RV. Avaliação do estado nutricional num contexto de mudanças socioeconômicas: o grupo indígena Suruí do Estado de Rondônia, Brasil. Cad Saude Publica 1991; 7(4):538562.

27. Carvalho NM, Giugliani ER, Seffrin CF, Hartmann RM. Seguimento de crianças com desnutrição moderada ou grave em população periférica (Brasil). Rev Saude Publica 1992; 26(4):223-228.

28. Sousa $A L$, Jardim PC, M onego ET, Raimundo MS, Lopes KE, Coelho J, Santos R, Gondim M R, Martins R, Daher GCF. Uma experiência multiprofissional na abordagem ao paciente hipertenso. Arq Bras Cardiol 1992; 59(1):31-35.

29. Torres MA, Sato K, Queiroz SS. Anemia em crianças menores de dois anos atendidas nas unidades básicas de saúde no Estado de São Paulo, Brasil. Rev Saude Publica 1994; 28(4):290-294.

30. Barros FC, Semer TC, Tonioli Filho S, Tomasi E, Victora CG. The impact of lactation centers on breastfeeding patterns, morbidity and growth: a birth cohort study. Acta Paediatr 1995; 84(11):1221-1226.

31. Cavalcante JW, Daza CM, Pessoa-Caval cante L, Pacheco WS, M enezes M G, M elo R, Gama CG. Prevalência e aspectos sócio-culturais e econômicos da hipertensão arterial em centro de saúde da zona norte de Manaus. Arq Bras Cardiol 1995; 65(6):493-496.

32. Capelli JCS, Anjos LA, Castro IRRC. Qualidade do valor da medida de massa corporal nos centros municipais do Rio de Janeiro, 1996. Cad Saude Publica 2002; 18(1):63-70.

33. Fernandes BS, Jerônimo ML, Leone C. Características familiares e cuidados e condições de saúde das crianças: seu papel no risco de desnutrição proteico-calórica. Pediatria 1996; 18(2):65-74.

34. Garcia-M ontrone V, Rose JC. U ma experiência educacional de incentivo ao aleitamento materno e estimulação do bebê, para mães de nível sócioeconômico baixo: estudo preliminar. Cad Saude Publica 1996; 12(1):61-68.

35. Puccini RF, Goihman S, Nóbrega FJ. Avaliação do programa de recuperação de desnutridos do município do Embu, na região metropolitana de São Paulo. J Pediatr 1996; 72(2):71-79.

36. Sala A, Nemes Filho A, Eluf Neto J. Avaliação da efetividade do controle da hipertensão arterial em unidade básica de saúde. Rev Saude Publica 1996; 30(2):161-167.

37. Lessa I, Fonseca J. Raça, aderência ao tratamento e/ou consultas e controle da hipertensão arterial. Arq Bras Cardiol 1997; 68(6):443-449.

38. Moura EFA. Duração do período de aleitamento materno de crianças atendidas em ambulatório de pediatria. J Pediatr 1997; 73(2):106-110.

39. Rodrigues CRM, M otta SS, Cordeiro AA, Lacerda EM A, Reichenhem ME. Prevalência e marcadores de risco associados em crianças entre 12 a 18 meses de idade atendidas nos ambulatórios do Instituto de Puericultura e Pediatria M artagão Gesteira. J Pediatr 1997; 73(3):189-194.
40. Almeida CAN, Ricco RG, Nogueira M PC, Ciampo $L A$, Rosa $F$. Prevalência de desnutrição em unidade básica de saúde da periferia de Ribeirão Preto (SP). 1998. Rev Paul Pediatr 1998; 16(4):185-1890.

41. Gutierrez MR, Bettiol H, Barbieri MA. Avaliação de um programa de suplementação alimentar. Rev Panam Salud Publica 1998; 4(1):32-39.

42. M onte CM, Ashworth A, Sa ML, Diniz RL. Effectiveness of nutrition centers in Ceará state, northeastern Brazil. Rev Panam Salud Publica 1998; 4(6):375-382.

43. Reichelt AJ, Spichler ER, Branchtein L, Nucci LB, Franco LJ, Schmidt MI. Fasting plasma glucose is a useful test for the detection of gestational diabetes. Brazilian Study of Gestational Diabetes (EBDG) Working Group. Diabetes Care 1998; 21(8):1246-1249.

44. Valverde MA, Patin RV, Oliveira FL, Lopez FA, Vitolo MR. Outcomes of obese children and adolescents enrolled in a multidisciplinary health program. Int J Obes Relat M etab Disord 1998; 22(6):513-519.

45. Ventura RN, Puccini RF, Silva EM K. Avaliação nutricional de crianças portadoras de "chiado no peito" atendidas nas unidades básicas de saúde do Jardim Santo Eduardo e do Jardim Santa Emília, Município do Embu no período de maio/88 a juIho/93. Rev Paul pediatr 1998; 16(4):201-207.

46. Almeida RM, M arins VM, Valle J. Breastfeeding, socio-economic conditions and nutritional status of children younger than 12 months in Brazil. Ann Trop Paediatr 1999; 19(3):257-262.

47. Araújo RB, Santos I, Cavaleti MA, Costa JSD, Béria JU. Avaliação do cuidado prestado a pacientes diabéticos em nível primário. Rev Saude Publica 1999; 33(1):24-32.

48. Boog MC. Dificuldades encontradas por médicos e enfermeiros na abordagem de problemas alimentares. Rev Nutr 1999; 12(3):261-272.

49. Boog MCF. Educação nutricional em serviços públicos de saúde. Cad Saude Publica 1999; 15(2):139-147.

50. Fujimori E, Oliveira IM, Cassana LM, Szarfarc SC. Estado nutricional del hierro de gestantes adolescentes, São Paulo, Brasil. Arch Latinoam Nutr 1999; 49(1):8-12.

51. Branchtein L, Schmidt MI, Matos MC, Yamashita T, Pousada JM, Duncan BB. Short stature and gestational diabetes in Brazil. Brazilian Gestational Diabetes Study Group. Diabetologia 2000; 43(7):848-851.

52. Montrone CVG, Arantes IS. Prevalência do aleitamento materno na cidade de São Carlos, São Paulo. J Pediatr 2000; 76(2):138-142.

53. Aguiar LG, Matos HJ, Brito Gomes M. Could fasting plasma glucose be used for screening high-risk outpatients for gestational diabetes mellitus? Diabetes Care 2001; 24(5):954-955

54. Assunção MC, Santos IS, Gigante DP. Atenção primária em diabetes no sul do país: estrutura, processo e resultado. Rev Saude Publica 2001; 35(1):88-95.

55. Batista Filho M, Ferreira LOC. U m modelo para avaliação rápida da situação nutricional e de saúde de crianças e mães no dia nacional de vacinação. Rev Bras Saúde M atern Infant 2001; 1(2):145-154.

56. Fujimori E, Cassana LM N, Szarfarc SC, Oliveira IMV, Guerra-Shinohara EM. Evolução do estado nutricional de grávidas atendidas na rede básica de saúde, Santo André, Brasil. Rev Latino-Am Enfermagem 2001; 9(3):64-68. 
57. Nucci LB, Duncan BB, Mengue SS, Branchtein L, Schmidt MI, Fleck ET. Assessment of weight gain during pregnancy in general prenatal care services in Brazil. Cad Saude Publica 2001; 17(6):1367-1374.

58. Nucci LB, Schmidt MI, Duncan BB, Fuchs SC, Fleck ET, Britto M M S. Nutritional status of pregnant women: prevalence and associated pregnancy outcomes. Rev Saude Publica 2001; 35(6):502-507.

59. Pierin AM G, Mion Jr D, Fukushima JT, Pinto AR, Kaminaga MM. O perfil de um grupo de pessoas hipertensas de acordo com conhecimento e gravidade da doença. Rev Esc Enf USP 2001; 35(1):11-18.

60. Santos I, Victora CG, M artines J, Goncalves H, Gigante DP, Valle NJ, Pelto G. Nutrition counseling increases weight gain among Brazilian children. J Nutr 2001; 31(11):2866-2873.

61. Schmidt MI, Duncan BB, Reichelt AJ, Branchtein L, M atos MC, Costa e Forti A, Spichler ER, Pousada JM, Teixeira M M, Yamashita T. Brazilian Gestational Diabetes Study Group. Gestational diabetes mellitus diagnosed with a 2-h 75-g oral glucose tolerance test and adverse pregnancy outcomes. Diabetes Care 2001; 24(7):1151-1155.

62. Alberti LR, Vasconcellos LS, Xavier CC, Werneck GAF. Avaliaçäo do estado nutricional de crianças de uma região atendida pelo programa de saúde da família em Itabira - M G. Rev M ed M inas Gerais 2002; 12(1):2-6

63. Assunção MC, Santos IS, Costa JSD. Clinical management of diabetic patients: process evaluation in Pelotas, Southern Brazil. Cad Saude Publica 2002; 18(1):205-211.

64. Brienza AM, M ishima SM, Frederico P, Clapis MJ. Grupo de reeducação alimentar: uma experiência holística em saúde na perspectiva familiar. Rev Bras Enferm 2002; 55(6):697-700.

65. Cesar JA, Cavaleti MA, Holthausen RS, Lima LG. $M$ udanças em indicadores de saúde infantil em um município com agentes comunitários: 0 caso de Itapirapuã Paulista, Vale do Ribeira, São Paulo, Brasil. Cad Saude Publica 2002; 18(6):1647-1654.

66. Hadler M CCM, Juliano Y, Sigulem DM. Anemia do lactente: etiologia e prevalência. J Pediatr 2002; 78(4):321-326.

67. Nascimento E, Souza SB. Avaliação da dieta de gestantes com sobrepeso. Rev Nutr 2002; 15(2):173-179.

68. Oliveira MIC, Camacho LAB. Impacto das unidades básicas de saúde na duração do aleitamento materno exclusivo. Rev Bras Epidemiol 2002; 5(1):41-51.

69. Silva DO, Recine EGIG, Queiroz EFO. Concepções de profissionais de saúde da atenção básica sobre a alimentação saudável no Distrito Federal, Brasil. Cad Saude Publica 2002; 18(5):1367-1377.

70. Venâncio SI, Escuder MML, Kitoko P, Rea MF, M onteiro CA. Freqüência e determinantes do aleitamento materno em municípios do Estado de São Paulo. Rev Saude Publica 2002; 36(3):313-318.

71. Azevedo DV, Sampaio HA. Consumo alimentar de gestantes adolescentes atendidas em serviço de assistência pré-natal. Rev Nutr 2003; 16(3):273-280.

72. Kac G, Benicio M HDA, Valente JG, VelasquezM elendez G. Postpartum weight retention among women in Rio de Janeiro: a follow-up study. Cad Saude Publica 2003; 19(Supl.):S149-S161.
73. Lessa AC, Devincenzi MU, Sigulem DM. Comparação da situação nutricional de crianças de baixa renda no segundo ano de vida, antes e apos a implantação de programa de atenção primaria à saúde. Cad Saude Publica 2003; 19(2):505-514.

74. Strelec MAAM, Pierin AM G, Mion Júnior D. A influência do conhecimento sobre a doença e a atitude frente à tomada de remédios no controle da hipertensão arterial. Arq Bras Cardiol 2003; 81(4):349-354.

75. Uchimura TT, Szarfarc SC, Latorre M RD, Uchimura NS, Souza SB. Anemia e peso ao nascer. Rev Saude Publica 2003; 37(4):397-403.

76. Vieira GO, Silva LR, Vieira TO. Alimentação infantil e morbidade por diarréia. J Pediatr 2003; 79(5): 449-454.

77. Vieira M LF, Silva JLCP, Barros-Filho AA. A amamentação e a alimentação complementar de filhos de mães adolescentes são diferentes das de filhos de mães adultas? J Pediatr 2003; 79(4):317-324.

78. Barros DC, Pereira RA, Gama SG, Leal MC. O consumo alimentar de gestantes adolescentes no $\mathrm{M} \mathrm{u}$ nicípio do Rio de Janeiro. Cad Saude Publica 2004; 20(Supl. 1):S121-S129.

79. Devincenzi MU, Colugnati FAB, Sigulem DM. Factores de protección para la anemia ferropriva: estudio prospectivo en niños de bajo nivel socioeconómico. Arch Latinoam Nutr 2004; 54(2):174-1749.

80. Hadler MC, Colugnati FA, Sigulem DM. Risks of anemia in infants according to dietary iron density and weight gain rate. Prev M ed 2004; 39(4):713-721.

81. Kac G, Benicio MHDA, Velasquez-M elendez G, Valente JG, Struchiner CJ. Breastfeeding and postpartum weight retention in a cohort of Brazilian women. Am J Clin Nutr 2004; 79(3):487-493.

82. Kac G, Benício MHDA, Velásquez-M eléndez G, Valente JG, Struchiner CJ. Gestational weight gain and prepregnancy weight influence postpartum weight retention in a cohort of Brazilian women. J Nutr 2004; 134(3):661-666.

83. Rotenberg S, Vargas S. Práticas alimentares e o cuidado da saúde: da alimentação da criança à alimentação da família. Rev bras saúde matern infant 2004; 4(1):85-94.

84. Souza Al, Batista Filho M, Ferreira LOC, Figueirôa JN. Efetividade de três esquemas com sulfato ferroso para tratamento de anemia em gestantes. Rev Panam Salud Pública 2004; 15(5):313-319.

85. Vieira GO, Silva LR, Vieira TO, Almeida JAG, Cabral VA. Hábitos alimentares de criancas menores de 1 ano amamentadas e não-amamentadas. J Pediatr 2004; 80(5):411-416.

86. Assunção MCF, Santos IS, Valle NCJ. Blood glucose control in diabetes patients seen in primary health care centers. Rev Saude Publica 2005; 39(2):183-190.

87. Batista MCR, Priore SE, Rosado LEFPL, Tinôco ALA, Franceschini SCC. Avaliação dos resultados da atenção multiprofissional sobre o controle glicêmico, perfil lipídico e estado nutricional de diabéticos atendidos em nível primário. Rev Nutr 2005; 18(2):219-128.

88. Coelho EB, Moysés N eto $M$, Palhares $R$, Cardoso MCM, Geleilete TJM, Nobre FN. Relação entre a assiduidade às consultas ambulatoriais e o controle da pressão arterial em pacientes hipertensos. Arq Bras Cardiol 2005; 85(3):157-161. 
89. Cuervo M RM, Aerts DRGC, Halpern R. Vigilância do estado nutricional das criancas de um distrito de saúde no Sul do Brasil. J Pediatr 2005; 81(4):325-331.

90. Georg AE, Duncan BB, Toscano CM, Schmidt MI, M engue $S$, Duarte $C$, Polanczyk CA. Analise econômica de programa para rastreamento do diabetes mellitus no Brasil. Rev Saude Publica 2005; 39(3):452-460

91. Mano GMP, Pierin AMG. Avaliação de pacientes hipertensos acompanhados pelo Programa Saúde da Família em um Centro de Saúde Escola. Acta Paul enferm 2005; 18(3):269-275.

92. Marques APO. Arruda IKG, Espírito Santo ACG, Guerra MD. Consumo alimentar em mulheres idosas com sobrepeso. Textos Envelhec 2005; 8(2):169186.

93. Neves M BP, Silva EM K, M orais M B. Prevalência e fatores associados a deficiência de ferro em lactentes atendidos em um centro de saúde-escola em Belém, Para, Brasil. Cad Saude Publica 2005; 21(6):1911-1918.

94. Oliveira MIC, Camacho LAB, Souza IEO. Promoção, proteção e apoio à amamentação na atenção primária à saúde no Estado do Rio de Janeiro, Brasil: uma política de saúde pública baseada em evidência. Cad Saude Publica 2005; 21(6):1901-1910.

95. Vieira GO, Vieira TO, Costa COM, Santana-Netto PV, Cabral VA. Uso do cartão da criança em Feira de Santana, Bahia. Rev Bras Saúde M atern Infant 2005; 5(2):177-384.

96. Andreto LM, Souza Al, Figueiroa JN, Cabral-Filho JE. Fatores associados ao ganho ponderal excessivo em gestantes atendidas em um serviço publico de prénatal na cidade de Recife, Pernambuco, Brasil. Cad Saude Publica 2006; 22(11):2401-2409.

97. Brunken GS, Silva SM, Franca GVA, Escuder M M, Venancio SI. Risk factors for early interruption of exclusive breastfeeding and late introduction of complementary foods among infants in midwestern Brazil. J Pediatr 2006; 82(6):445-451.

98. Castro MB, Kac G, Sichieri R. Padrão de consumo alimentar em mulheres no pós-parto atendidas em um centro municipal de saúde do Rio de Janeiro, Brasil. Cad Saude Publica 2006; 22(6):1159-1170.

99. Picoli RP, Carandina L, Ribas DLB. Saúde materno-infantil e nutrição de crianças Kaiowa e Guarani, Área Indígena de Caarapó, M ato Grosso do Sul, Brasil. Cad Saude Publica 2006; 22(1):223-227.

100. Piovesana PM, Colombo RCR, Gallani MCBJ. $\mathrm{H} y$ pertensive patients and risk factors related to physical activity and nutrition. Rev Gaucha Enferm 2006; 27(4):557-563.

101. Santos LA, M amede FV, Clapis MJ, Bernardi JVB. Nutritional guidance during prenatal care in public health services in Ribeirao Preto: discourse and care practice. Rev Lat Am Enfermagem 2006; 14(5):688-694.

102. Amado TC, Arruda IK, Ferreira RA. Aspectos alimentares, nutricionais e de saude de idosas atendidas no Núcleo de Atenção ao Idoso-NAI, Recife/ 2005. Arch Latinoam Nutr 2007; 57(4):366-372.

103. Araújo JC, Guimarães AC. Controle da hipertensão arterial em uma unidade de saúde da família. Rev Saude Publica 2007; 41(3):368-374.
104. Bustamante V, Trad LAB. Cuidando da saúde de crianças pequenas no contexto familiar: um estudo etnográfico com famílias de camadas populares. Cien Saude Colet 2007; 12(5):1175-1184.

105. Gastal DA, Pinheiro RT, Vazquez DP. Self-efficacy scale for Brazilians with type 1 diabetes. Sao Paulo M ed J 2007; 125(2):96-101.

106. Lacerda EM A, Kac G, Braga da Cunha C, Leal MC. Consumo alimentar na gestação e no pós-parto segundo cor da pele no município do Rio de Janeiro. Rev Saude Publica 2007; 41(6):985-994.

107. Martins M C, Oliveira YP, Coitinho DC, Santos LM P. Panorama das ações de controle da deficiência de vitamina A no Brasil. Rev Nutr 2007; 22(1):5-18.

108. Melo ASO, Assunção PL, Gondim SSR, Carvalho DF, Amorim MMR, Benício MHDA, Cardoso MAA. Estado nutricional materno, ganho de peso gestacional e peso ao nascer. Rev Bras Epidemiol 2007; 10(2):249-257.

109. Nascimento AL, Diniz AS, Arruda IKG. Deficiência de vitamina $A$ em idosos do Programa de Saúde da Família de Camaragibe, PE, Brasil. Arch Latinoam Nutr 2007; 57(3):213-218.

110. Silva DG, Priore SE, Franceschini SCC. Risk factors for anemia in infants assisted by public health services: the importance of feeding practices and iron supplementation. J Pediatr 2007; 83(2):149-156.

111. Silva SM, Brunken GS, Franca GVA, Escuder M M, Venancio SI. Evolução do aleitamento materno em uma capital da Região Centro-O este do Brasil entre 1999 e 2004. Cad Saude Publica 2007; 23(7): 1539-1546.

112. Stulbath TE, Benício MHDA, Andrezza R, Kono S. Determinantes do ganho ponderal excessivo durante a gestação. Rev Bras Epidemiol 2007; 10(1):99108.

113. Alves JG, Gale CR, Souza E, Batty GD. Effect of physical exercise on bodyweight in overweight children: a randomized controlled trial in a Brazilian slum. Cad Saude Publica 2008; 24(Supl. 2):S353-S359.

114. Carvalho MF, Lira PIC, Romani SAM, Santos IS, Veras AACA, Batista Filho M. Acompanhamento do crescimento em crianças menores de um ano: situação nos serviços de saúde em Pernambuco, Brasil. Cad Saude Publica 2008; 24(3):675-685.

115. Cesar JA, M endoza-Sassi RA, UImi EF, Dall'Agnol MM, Neumann NA. Diferentes estrategias de visita domiciliar e seus efeitos sobre a assistencia prenatal no extremo Sul do Brasil. Cad Saude Publica 2008; 24(11):2614-2622.

116. Coqueiro RS, Nery AA, Cruz ZV, Couto de Sá CK. Fatores associados ao sobrepeso em adultos acompanhados por uma unidade de saúde da família. Rev bras cineantropom desempenho hum 2008; 10(2):149-154

117. Engstrom EM, Castro IRR, Portela M, Cardoso LO, Monteiro CA. Effectiveness of daily and weekly iron supplementation in the prevention of anemia in infants. Rev Saude Publica. 2008; 42(5): 786-795.

118. Lagacci MT, Verussa TL, Vieira VG, U eno M, Cocetti M, Ceolim M F, Lima M HM. Estado nutricional de pacientes diabéticos atendidos em uma unidade básica de saúde, Campinas-SP. Cogitare Enferm 2008; 13(2):206-211. 
119. M olena-Fernandes CA, Carolino IDR, Elias RGM, $\mathrm{N}$ ardo Junior $\mathrm{N}$. Efeito do exercício físico aeróbio sobre o perfil lipídico de pacientes idosas, portadoras de Diabetes M ellitus tipo 2, atendidas em Unidade Básica de Saúde, M aringá, Estado do Paraná. Rev bras geriatr gerontol 2008; 11(2):167-180.

120. Rodrigues PL, Lacerda EMA, Schlussel MM, Spyrides $M H C, K a c G$. Determinants of weight gain in pregnant women attending a public prenatal care facility in Rio de Janeiro, Brazil: a prospective study, 2005-2007. Cad Saude Publica 2008; 24(Supl.2):S272-S284.

121. Santos LM P, Paes-Sousa R, Silva Junior JB, Victora CG. National Immunization Day: a strategy to monitor health and nutrition indicators. Bull World H ealth Organ 2008; 86(6):474-479.

122. Shibukawa AF, Silva EM K, Ichiki WA, Strufaldi MWL, Puccini RF. Prophylaxis for iron deficiency anemia using ferrous sulfate among infants followed up at a primary healthcare unit in the municipality of Embu-SP (2003/2004). São Paulo M ed J 2008; 126(2):96-101.

123. Baldissera VDA, Carvalho MDB, Pelloso SM. Adesão ao tratamento não-farmacológico entre hipertensos de um centro de saúde escola. Rev Gaúcha Enferm 2009; 30(1):27-32.

124. Bohlke M, Dullius F, M enezes J, Cunha T, Boemer $\mathrm{H}$, Menezes $\mathrm{A}$, Caruso P. Predictors of higher blood pressure in a clinical setting in normotensive children: a prospective study. Kidney Blood Press Res 2009; 32(3):205-209.

125. Buss C, Nunes M A, Camey S, Manzolli P, Soares RM, Drehmer M, Giacomello A, Duncan BB, Schmidt MI. Dietary fibre intake of pregnant women attending general practices in southern Brazil the ECCAGE Study. Public Health Nutr 2009; 12(9):1392-1398.

126. Dal Pizzol TS, Giugliani ERJ, M engue SS. Associação entre 0 uso de sais de ferro durante a gestação e nascimento pré-termo, baixo peso ao nascer e muito baixo peso ao nascer. Cad Saude Publica 2009; 25(1):160-168.

127. Ferreira CLRA, Ferreira M G. Características epidemiológicas de pacientes diabéticos da rede pública de saúde: análise a partir do sistema HiperDia. Arq Bras endocrinol metab 2009; 3(1):80-86.

128. Silva M M, Rocha L, Silva SO. Enfermagem em puericultura: unindo metodologias assistenciais para promover a saúde nutricional da criança. Rev Gaúcha Enferm 2009; 30(1):141-144.
129. Soares RM, Nunes MA, Schmidt MI, Giacomello A, Manzolli P, Camey S, Buss C, Drehmer M, Melere $C$, H offman J, Ozcariz S, Manenti CN, Pinheiro AP, Duncan BB. Inappropriate eating behaviors during pregnancy: prevalence and associated factors among pregnant women attending primary care in southern Brazil. Int J Eat Disord 2009; 42(5):387-393.

130. Drehmer M, Camey S, Schmidt MI, Olinto MT, Giacomello A, Buss C, M elere C, Hoffmann J, Manzolli P, Soares RM, Ozcariz S, Nunes M A. Socioeconomic, demographic and nutritional factors associated with maternal weight gain in general practices in Southern Brazil. Cad Saude Publica 2010; 26(5):1024-1034.

131. Lemos JO, Rondo PH, Pereira JA, Oliveira RG Freire $M B$, Sonsin $\mathrm{PB}$. The relationship between birth weight and insulin resistance in childhood. Br J Nutr 2010; 103(3):386-392.

132. Pimentel GD, Portero-McLellan KC, Oliveira EP, Spada AP, O shiiwa M, Zemdegs JC, Barbalho SM. Long-term nutrition education reduces several risk factors for type 2 diabetes mellitus in Brazilians with impaired glucose tolerance. Nutr Res 2010; 30(3):186-190.

133. Silva LMC, Palha PF, Barbosa G, Rodrigues Protti ST, Ramos AS. Aposentados com diabetes tipo 2 na Saúde da Família em Ribeirão Preto, São Paulo Brasil. Rev Esc Enferm USP 2010; 44(2):462-468.

134. Batista Filho M, Rissin A. Deficiências nutricionais: ações específicas do setor saúde para o seu controle. Cad Saude Publica 1993; 9(2):130-135.

135. Batista Filho M, Ferreira LOC. Prevenção e tratamento da anemia nutricional ferropriva: novos enfoques e perspectivas. Cad Saude Publica 1996; 12(13):411-415.

136. Assis AM O, Santos SM C, Freitas MCS, Santos JM Silva M CM . O programa saúde da família: contribuições para uma reflexão sobre a inserção do nutricionista na equipe multidisciplinar. Rev Nutr 2002; 15(3):255-266.

137. Coutinho JG, Gentil PC, Toral N. A desnutrição e obesidade no Brasil: o enfrentamento com base na agenda única da nutrição. Cad Saude Publica 2008; 24(Supl. 2):S332-S340. 